\title{
Modelling Down syndrome
}

\section{Frank Buckley}

Animal models are extensively used in genetics, neuroscience and biomedical research. Recent studies illustrate the usefulness and the challenges of research utilising genetically engineered mice to explore the developmental biology of Down syndrome. These studies highlight many of the issues at the centre of what we understand about Down syndrome, and may one day point to useful ways to improve quality of life for people living with Down syndrome.

All people diagnosed with Down syndrome have an extra copy of at least part of chromosome 21 in at least some of their cells. Most have a complete additional copy of chromosome 21 in every cell (BOX 1). These extra copies of genes, present from the point of conception, begin a complex cascade of consequences that depend (among other things) on the additional genes from chromosome 21, other genes on other chromosomes, anatomical location, developmental progress and the environment. The precise details of how these processes work and interact are not clearly understood. Such questions lie at the heart of modern genetics and cognitive neuroscience research.

\section{Complex systems}

The molecular biology of just a single cell is complicated (FIGURE 1) and modelling complex systems is difficult ${ }^{[1,2]}$. Describing how disruptions in gene 'doses' at the cellular level in people with Down syndrome contribute to (for example) particular difficulties in verbal short-term memory ${ }^{[3]}$ or comparatively slower progress in language development than reading development ${ }^{[4]}$ may seem an insurmountable challenge. Yet, this is the ultimate goal of much of the research into the genetics, molecular biology and neuroscience of Down syndrome.

\section{Gene 'dosage'}

The underlying hypothesis of most research into the developmental biology of Down syndrome is that the additional copies of genes from chromosome 21 lead to the additional production of certain molecules (the proteins encoded by these

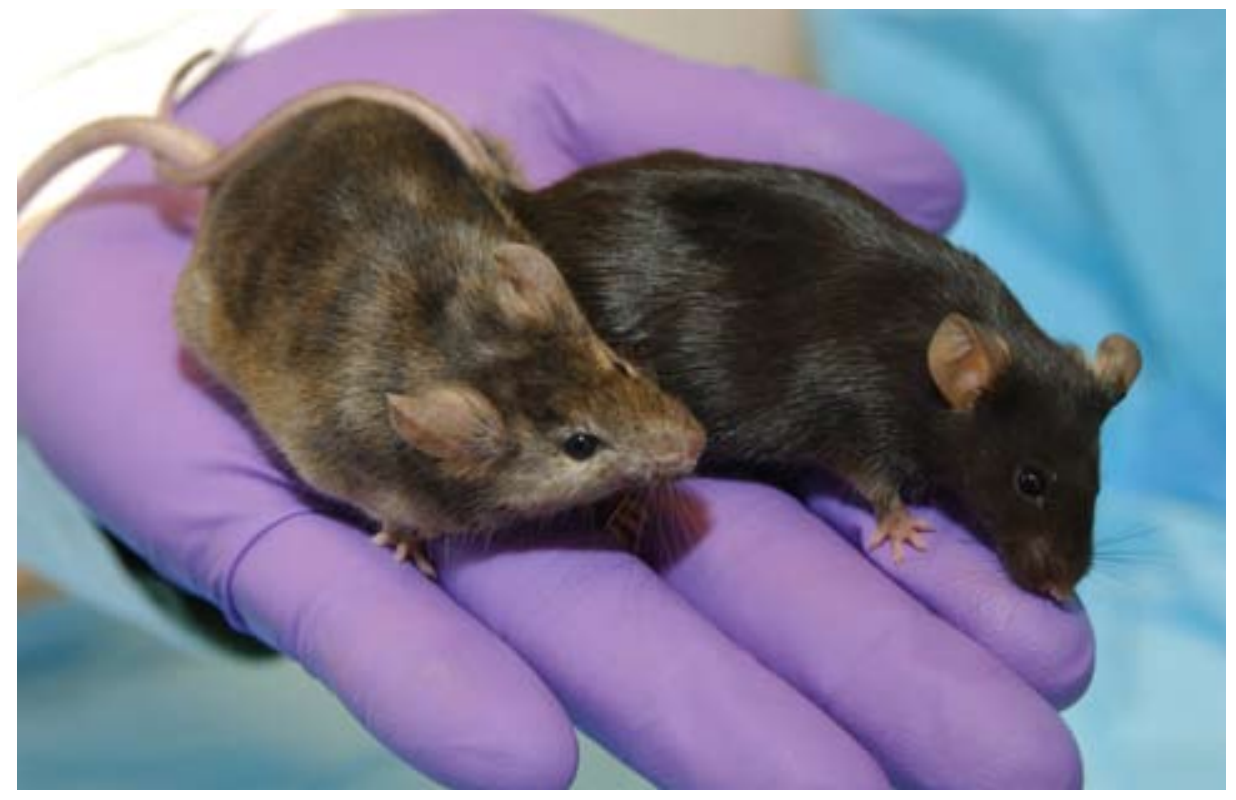

The organism of choice for much of modern biomedical research is the mouse. Several genetically engineered strains have been made to study the biology of Down syndrome.

Box 1 | The genetics of Down syndrome

Approximately $95 \%$ of people with Down syndrome have an additional copy of a whole chromosome 21 in every cell (trisomy 21). Around 3\% of people with Down syndrome have an additional copy of a stretch of chromosome 21 in every cell (translocation or partial trisomy 21). Among the remaining $2 \%$ of people with Down syndrome, some cells have the additional copy of chromosome 21 and some do not (mosaic Down syndrome).

\section{FURTHER READING}

Leshin L. Trisomy 21: The story of Down syndrome. Down Syndrome Health Issues. 2003. Available from: http://www.ds-health.com/trisomy.htm

Leshin L. Mosaic Down syndrome. Down Syndrome Health Issues. 2000. Available from: http://www.ds-health.com/mosaic.htm

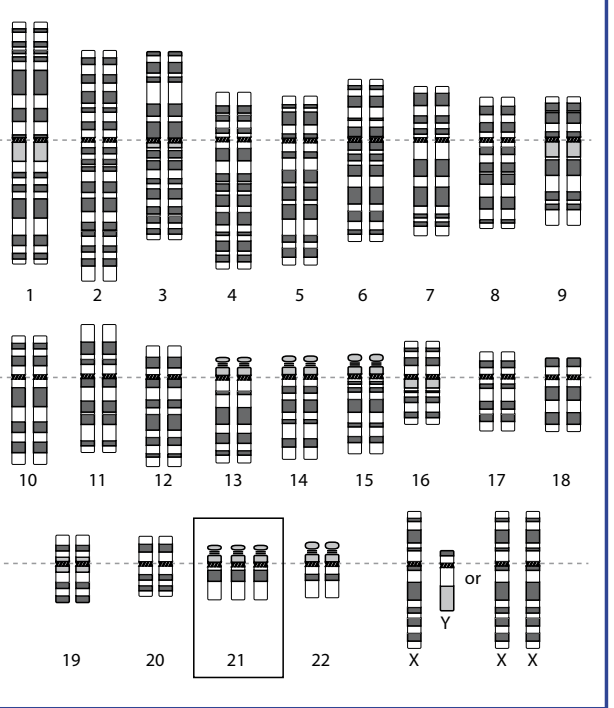


genes) and that this 'over-expression' more or less leads to many of the features commonly observed among people with Down syndrome ${ }^{[5,6]}$. In the simplest case, the product of a single gene on chromosome 21 might directly contribute to a specific feature. Perhaps more often, it may be that multiple genes (on chromosome 21 and other chromosomes) interact to influence a particular trait ${ }^{[6]}$.

It is hoped that a better understanding of these molecular pathways will inform the development of effective gene or pharmacological therapies for at least some aspects of Down syndrome. A better understanding of how these processes influence development - particularly brain development - may also inform our understanding of how some cognitive processes are disrupted for people with Down syndrome and thereby inform effective educational practice.

\section{Common ancestors}

Modern human beings' genomes are the product of approximately 4 billion years of evolution. Humans and chimpanzees last shared a relative somewhere around 6 million years ago and we parted company with an ancestor shared with mice some 75 million years ago. However, over $90 \%$ of the DNA sequences of monkeys, mice and people are similar ${ }^{[7,8]}$.

In a study published in $2006^{[10]}$, Katheleen Gardiner and Alberto Costa reviewed what is understood about the genes on human chromosome 21, similar genes in mice and reported a systematic search for comparable genes in nine other organisms from yeast to chimpanzee. Out of a total of approximately 400 genes on human chromosome 21, the authors identified 26 similar genes conserved as far back as yeast, including genes that appear to be involved in growth and DNA replication, noting that several of them are lethal when removed ('knocked out') from yeast. Further similar genes were identified in fish, insects, birds and mammals.

Gardiner and Costa note that where similar genes retain their ancestral function, they may help to predict the functions of genes on human chromosome 21. However, genes do not function in isolation and, as the authors note, the functions identified by knockout studies in simpler organisms may not directly relate to the consequences of an approximate

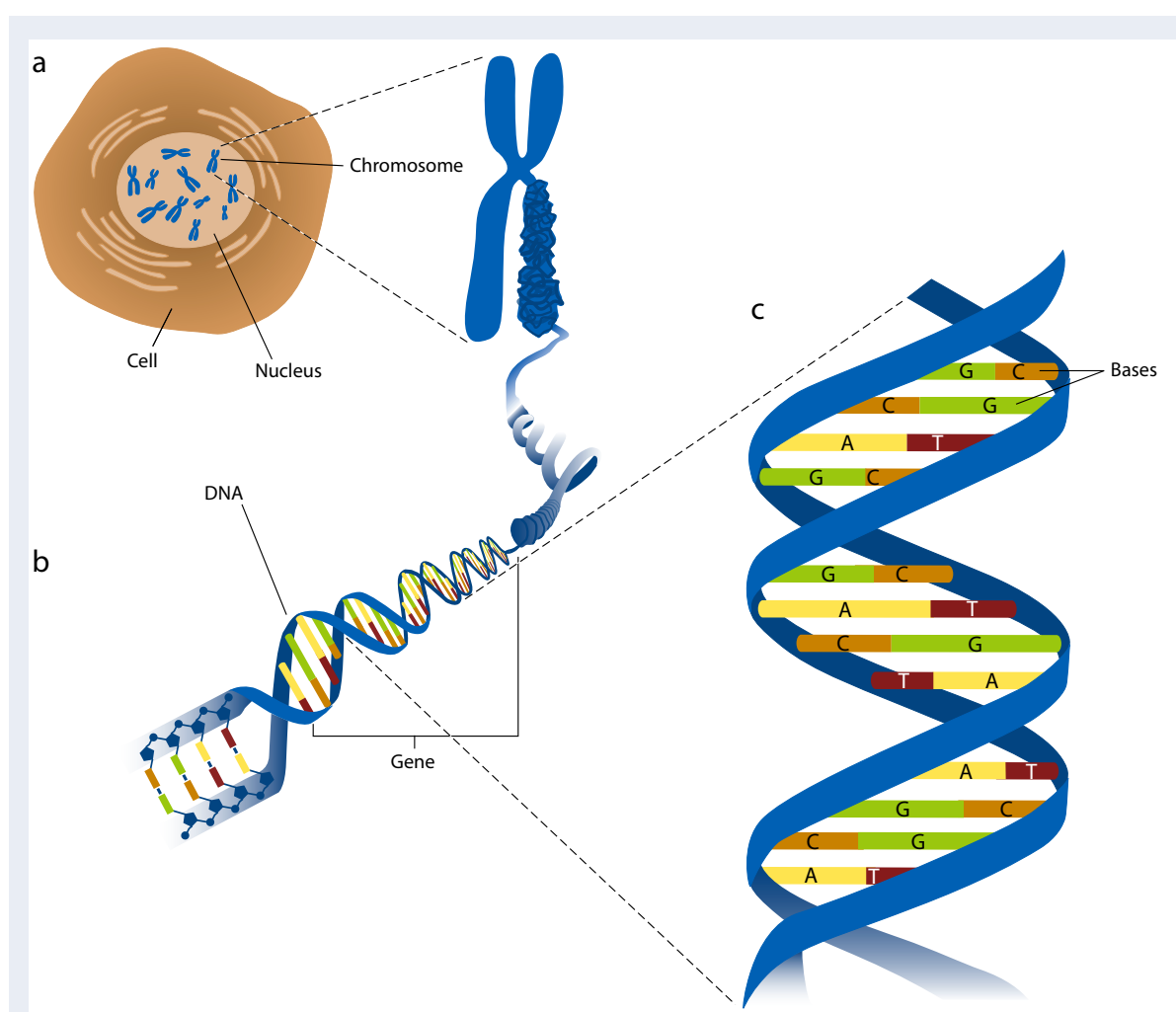

Figure 1 | Life at many levels a Every cell carries a copy of the 'recipe' for the organism, encoded by DNA, long strands of which are wrapped up into chromosomes. $\mathbf{b}$ A gene is a stretch of DNA that contains the 'instructions' for how and when to make certain molecules (proteins). c DNA is a long chain of molecules grouped into base pairs. The human genome records some 3 billion DNA base pairs that (among other things) carry the 'instructions' for making some 100,000 molecules that work together to make up the various tissues, organs and other features of our anatomy. A human being possesses around 100 trillion cells, many functioning in markedly different ways to fulfil different roles in different parts of the body. Of these cells, around 100 billion are nerve cells in the brain (neurons), each making perhaps several thousand connections to other neurons.

$50 \%$ over-expression in human Down syndrome. To explore these issues, model organisms that carry additional copies of genes that are similar to those found on human chromosome 21 are necessary.

\section{Model organisms}

Given our shared ancestry and considerable molecular similarity, other organisms make very useful experimental subjects for the study of genetics, molecular biology and neuroscience. Clearly, there are many biological experiments that cannot be carried out with living people - for both practical and ethical reasons.

Many organisms have been, and continue to be, studied in depth. However, the organism of choice for much of modern biomedical research is the mouse ${ }^{[11-13]}$. The mouse genome has been sequenced ${ }^{[8]}$ and, recently, a detailed map of where different genes are active (expressed) in the mouse brain has been completed ${ }^{[14]}$. Mice can be genetically altered, bred easily and may be readily dissected and studied at any stage of development.

The first link between human chromosome 21 and mouse chromosome 16 was established in 1979 and soon after mice that carried an extra copy of mouse chromosome 16 (referred to as Ts16) were identified as a potential model for the study of Down syndrome ${ }^{[5]}$.

As well as carrying additional copies of comparable genes, an animal model should also display features that are comparable to those observed among people with Down syndrome. Ts16 embryos do show a number of anatomical similarities to human embryos with Down syndrome $^{[15]}$, but usually do not survive past birth and so their behaviour cannot be studied $^{[5,15]}$. Since only parts of mouse chromosome 16 correspond to parts of human chromosome $21^{[\text {REF 16] }}$ and an extra copy of the whole of mouse chromosome 16 is present in Ts16 mice, they also carry extra copies of genes not present in human 
trisomy 21 and it is possible that these contribute to the features observed.

A closer genetic match is provided by mouse models that are trisomic for only a part of mouse chromosome 16. The Ts65Dn mouse has been extensively studied since the early 1990s. The Ts65Dn mice carry an additional copy of a part of mouse chromosome 16 that is similar to a part of human chromosome 21. Ts65Dn exhibit a number of features that appear to be comparable to aspects of human Down syndrome, including some types of learning and memory difficulties, neuroanatomical characteristics and a lower life expectancy ${ }^{[5,15]}$. However, Ts65Dn mice do not carry extra copies of all of the segments on mouse chromosome 16 that are syntenic to segments on human chromosome 21. They also carry an additional copy of part of mouse chromosome 17 that is not comparable to any part of human chromosome 21.

Last year Zhongyou Li, Eugene $\mathrm{Yu}$ and colleagues reported that they had created mice carrying an extra copy of all of the segments on mouse chromosome 16 that are syntenic to human chromosome $21^{\text {[REF 17] }}$. In contrast to Ts65Dn mice, these new $\mathrm{Dp}(16) 1 Y u$ mice carry extra copies of a larger region of comparable DNA from mouse chromosome 16, and do not carry additional copies of part of mouse chromosome 17. In theory, therefore, they may be expected to be a more accurate model of human Down syndrome. Li and colleagues report that heart defects are common among the $\mathrm{Dp}(16) 1 \mathrm{Yu}$ mice, comparable to those observed in human Down syndrome. It has recently been reported that the $\mathrm{Dp}(16) 1 \mathrm{Yu}$ mice show some learning and memory difficulties.

\section{Exploring specifics}

While $\mathrm{Li}$ and colleagues have reported a mouse with extra copies of more comparable genes, Lisa Olson and colleagues have reported a study of the brains and behaviour of mice that are trisomic for a much smaller region of mouse chromosome 16 than Ts65Dn mice ${ }^{[18]}$. Olson and colleagues previously reported that the skulls of these Ts1Rhr mice did not show growth patterns similar to those observed in human Down syndrome ${ }^{[19]}$. So why continue to study a mouse with fewer similar genes?

Studies of small numbers of people carrying extra copies of only part of chromosome 21 (partial trisomy) in the 1970s and 1980s suggested that only parts of the chromosome were necessary to lead to certain features associated with Down syndrome. The region encompassing these parts became known as the 'Down Syndrome Critical Region' (DSCR) ${ }^{[5,19]}$. The Ts1Rhr mouse, studied by Olson and colleagues is trisomic only for the region of mouse chromosome 16 that is comparable to the DSCR. Along with a mouse that has one instead of the usual two copies of the same region (segmentally monosomic Ms1Rhr mice), the Ts1Rhr mouse permits the study of the effects of increased gene 'doses' in this particular segment of the genome.

Studying Ts1Rhr, Ts65Dn and Ms1Rhr mice, Olson and colleagues report that the genes found in this part of mouse chromosome 16 are not alone sufficient to lead to lower performance on a test of spatial memory in rodents: The Ts1Rhr mice (with only the 'critical' region) performed just as well as closely related 'typical' mice ${ }^{[18]}$, whereas Ts65Dn mice (with extra copies of genes additional to those in the 'critical' region) perform worse than their typical littermates.

\section{Genes, brains and behaviour}

If multiples genes interact to contribute to the features of Down syndrome in ways that are not easy to identify in 'simple' gene knockout studies and do not lie neatly in a 'critical region', then perhaps the study of different mouse models, trisomic for different genes may shed light on these complex molecular pathways. Two further recent studies suggest this approach might be useful. Fabian Fernandez and Craig Garner have recently reported a study of memory function in Ts65Dn and Ts1Cje mice. Ts1Cje mice are trisomic for around $75 \%$ of the genes triplicated in Ts65Dn mice ${ }^{[20]}$. They therefore offer a model for contrasting the effects of over-expression of some genes in the Ts65Dn mouse.

Fernandez and Garner report that the Ts1Cje mice do not display difficulties on measures of short and long term object memory. They contrast this finding with evidence that Ts65Dn mice have little difficulty with short term object recognition tests, yet find object recognition more problematic over longer periods of time. The authors suggest that these differences may be indicative of the extent to which the hippocampus is functionally altered. They suggest that this study adds further support to the theory that the function of the hippocampus plays a central role in some of the learning difficulties observed among people with Down syndrome.

Not only differing memory abilities can be observed among mice with different trisomic genes. Two further recent studies explore brain shape, size and skull growth in various mouse models.

Kristina Aldridge and colleagues have reported a study of the brains of Ts65Dn mice and Ts1Rhr mice using high resolution magnetic resonance images (MRIs) ${ }^{[21]}$. They found differencesin brain volumeand shape between the Ts1Rhr mice (trisomic for relatively few genes in the so-called critical region) and the Ts65Dn (trisomic for more genes). Noting that each mouse 
model exhibited anatomical features that could be paralleled to features observed in human Down syndrome, but that the features were different in each model, the authors argue that this is further evidence that gene 'doses' in the so-called critical region cannot alone be responsible for all aspects of Down syndrome.

Cheryl Hill and colleagues have recently reported a study of skull growth in Ts65Dn mice ${ }^{[22]}$. Examining key features in newborn and adult Ts65Dn mice and comparing them with typical littermates, Hill and colleagues report that anatomical features associated with human Down syndrome are found in newborn Ts65Dn mice and that some of these features continue to develop differently from typical littermates through to adulthood. The authors emphasise growth is an iterative process, with genes governing developing structures that in turn effect future gene expression (and so on). They argue that studies of different mouse models (trisomic for different sets of genes) are needed to explore these complex, developmental interactions.

\section{Individuality}

It is often noted that the features of Down syndrome are highly variable among individuals - perhaps more so than the general population. Although more common, not all people with Down syndrome are born with heart defects, develop hypothyroidism or obstructive sleep apnoea, or exhibit behavioural difficulties. The range of ability observed among people with Down syndrome on many cognitive measures is wide ${ }^{[4,23]}$. It is not clear to what extent this variability is the result of variations in upbringing and environment, to what extent it is due to individual genotype and to what extent it is due to 'general' effects of trisomy 21.

One prediction of the gene 'dosage' hypothesis is that there will be $50 \%$ more of the 'products' of genes present in three copies than is the case when the usual two copies are present. Studies of mouse models and some human tissue samples seem to generally support this prediction. However, if the 'disruption' is so uniform, then how does variability in the fea- tures of Down syndrome arise?

Marc Sultan and colleagues have recently investigated the 'products' of genes (expression levels) that are triplicated in different parts of the brain of Ts65Dn mice. Unlike previous studies that have reported pooled samples (from multiple individuals), Sultan and colleagues analyse individual variations in expression levels in three areas of the brain ${ }^{[24]}$. The authors confirm that pooled samples show around $50 \%$ over-expression of a measure of gene expression, but go on to show some variation between individuals. The authors note that the expression levels of most of the genes vary by around $20 \%$ $50 \%$, although a few are much more variable and some are much less variable. The authors suggest that those that are much less variable may be good candidates for features of Down syndrome that are more constant among individuals.

It is perhaps worth noting that the Ts65Dn mice are bred with a cross from two strains of mice and that the genetic diversity among individuals may not be as great as among typical populations. The mice are also housed in modest, tightly controlled conditions to minimise environmental differences across experimental animal subjects. Therefore, it might be expected that typical variation in gene expression between individuals is more than that observed among the experimental mice.

It seems clear that better healthcare and richer, inclusive social and educational environments are helping people with Down syndrome to achieve more. Given this and given that improved cognitive function and neuroanatomical changes have been observed in mice given more stimulation (environmental enrichment), it might seem surprising that relatively few studies have investigated the effects of enriched environments on mice designed to model Down syndrome ${ }^{[25]}$. How important environment is in modulating the effects of trisomy in these animals is therefore not clear. Identifying the effects of environmental changes and comparing them to those of other possible therapeutic interventions is an important theoretical and practical issue.

\section{The best-laid schemes o' mice an' men}

Fernandez and Garner emphasise evidence for difficulties observed among people with Down syndrome that suggest hippocampal dysfunction ${ }^{[26]}$ comparable to that observed in mouse models. Aldridge and colleagues draw parallels between anatomical features observed in mouse models and people with Down syndrome. Hill and colleagues also point out that their study of skull growth shows clear parallels to features observed among people with Down syndrome and that this validates the use of animal models to explore how altered gene 'doses' disrupt molecular pathways and effect development.

So what do these studies of genetically altered mice tell us about human development and Down syndrome? It is not easy to answer this question, yet. Clearly, mice do not speak and will likely be of limited use in exploring the neurological factors contributing to the language processing abilities of people with Down syndrome. The extent to which memory and learning are directly comparable between mice and humans still remains to be determined.

However, despite limitations, these animal models are providing lots of useful insights into the molecular biology, anatomy and neuroscience of Down syndrome that may lead to useful therapeutics ${ }^{[27]}$.

As Fernandez and Garner note ${ }^{[20]}$, more work is required to compare the specific nature of the difficulties observed in mouse models with those observed in humans. It is often commented that accurate, detailed and quantified descriptions of the features 
of Down syndrome (the phenotype) are vital for informed analysis of genotypephenotype links ${ }^{[5,6]}$. Indeed, given the age of some studies of people with Down syndrome, there is a case to be made for a detailed reappraisal of some of what we understand about the Down syndrome phenotype ${ }^{[28]}$. The sooner further work in both men and mice is progressed, the sooner we will be able to better identify further ways to assist people with Down syndrome.

\section{Acknowledgements}

The author would like to thank David Patterson for helpful comments on drafts of this article.

Frank Buckley is at Down Syndrome Education International. e-mail: frank.buckley@downsed.org

doi: 10.3104/updates.2054

19. Olson LE, Richtsmeier JT, Leszl J, Reeves RH A chromosome 21 critical region does not cause specific Down syndrome phenotypes. Science. 2004;306(5696):687-90. doi:10.1126/science.1098992

20. Fernandez F, Garner CC. Object recognition memory is conserved in $\mathrm{Ts} 1 \mathrm{Cje}$, a mouse model of Down syndrome. Neuroscience Letters. 2007;421(2):137-41.

21. Aldridge $\mathrm{K}$, Reeves $\mathrm{RH}$, Olson LE, Richtsmeier JT. Differential effects of trisomy on brain shape and volume in related aneuploid mouse models. America Journal of Medical Genetics Part A. 2007;143(10):1060-70. doi:10.1002/ajmg.a.31721

22. Hill CA, Reeves RH, Richtsmeier JT. Effects of aneuploidy on skull growth in a mouse model of Down syndrome. Journal of Anatomy. 2007;210(4):394-405.

23. Groen MA, Laws G, Nation K, Bishop DVM. A case of exceptional reading accuracy in a child with Down syndrome: Underlying skills and the relation to reading comprehension. Cognitive Neuropsychology. 2006;23(8):1190-1214. doi:10.10 80/02643290600787721

24. Sultan M, Piccini I, Balzereit D, Herwig $R$, Saran $\mathrm{NG}$, Lehrach H, Reeves RH, Yaspo ML. Gene expression variation in Down's syndrome mice allows prioritization of candidate genes. Genome Biology. 2007;8(5):R91 doi:10.1186/gb2007-8-5-r91

25. Nithianantharajah J, Hannan AJ. Enriched environments, experience-dependent plasticity and disorders of the nervous system. Nature Reviews Neuroscience. 2006;7:697-709. doi:10.1038/ nrn1970

26. Pennington BF, Moon J, Edgin J, Stedron J, Nadel L. The neuropsychology of Down syndrome: evidence for hippocampal dysfunction. Child Development. 2003;74(1):75-93. doi:10.1111/14678624.00522

27. Fernandez F, Morishita W, Zuniga E, Nguyen J Blank M, Malenka RC, Garner CC. Pharmacotherapy for cognitive impairment in a mouse model of Down syndrome. Nature Neuroscience. 2007;10(4);411-3. doi:10.1038/nn1860

28. Nadel L. Down's syndrome: a genetic disorder in biobehavioral perspective. Genes Brain and Behavior. 2003;2(3):156-66. doi:10.1034/j.1601183X.2003.00026.X 